\title{
DETERMINATION OF THE EXTREME VALUES OF THE SPECTRUM OF A BOUNDED SELF-ADJOINT OPERATOR ${ }^{1}$
}

\author{
WILLIAM KARUSH
}

1. Introduction. Let $A$ be a bounded self-adjoint operator on a Hilbert space $R$ with spectral family of projections $F(\lambda)$. Let $\lambda_{1}^{\prime}$ be the supremum of the spectrum $\mathrm{S}(A)$ of $A$. We consider an iterative procedure for the determination of $\lambda_{1}^{\prime}$ and, in case $\lambda_{1}^{\prime}$ is a characteristic number, for the determination of a characteristic vector belonging to $\lambda_{1}^{\prime}$. The procedure involves the solution of a characteristic value problem of finite dimension at each step of the iteration, this dimension being the same for each step and fixed at a convenient value at the outset.

The method is based upon the observation that with

$$
\mu(x)=\frac{(x, A x)}{(x, x)}, \quad x \neq 0 \text { in } R,
$$

we have

$$
\lambda_{1}^{\prime}=\sup _{x \neq 0} \mu(x), \quad x \text { in } R .
$$

The iteration is determined as follows. Select an integer $s>1$ and an initial vector $x^{0}$ in $R$. In the space $A\left(x^{0}\right)$ spanned by the vectors $x^{0}, A x^{0}, \cdots, A^{n-1} x^{0}$ determine a vector $x^{1}$ which maximizes $\mu(z)$ for $x$ in $A\left(x^{0}\right)$; we shall show that $x^{1}$ is unique, apart from a scalar factor, and that it may be chosen in the form $x^{1}=x^{0}+\eta$, with $\left(x^{0}, \eta\right)$ $=0$. Clearly $\mu\left(x^{0}\right) \leqq \mu\left(x^{1}\right)$. In the next step we similarly choose $x^{2}=x^{1}$ $+\eta$ as the vector in the space $A\left(x^{1}\right)$ spanned by $x^{1}, A x^{1}, \cdots, A^{{ }^{-1}} x^{1}$ which maximizes $\mu(x)$ in this space. In this way we construct a sequence $\left\{x^{i}\right\}$ with nondecreasing values $\mu\left(x^{i}\right)$. The determination of each $x^{i}$ involves solving for a characteristic vector of a self-adjoint matrix of order at most $s .^{2}$

Now suppose $x^{0}$ is such that $F(\lambda) x^{0} \neq x^{0}$ for $\lambda<\lambda_{1}^{\prime}$. Then we shall prove that the numbers $\mu\left(x^{i}\right)$ converge to $\lambda_{1}^{\prime}$; and, further, that the unit vectors $u^{i}=x^{i} /\left|x^{i}\right|$ converge weakly to a characteristic vector

Received by the editors December 22, 1950.

1 The preparation of this paper was sponsored (in part) by the Office of Naval Research.

${ }^{2}$ An iterative procedure different from ours is given by $x^{i+1}=A x^{i}$. For a discussion of this method see R. Wavre, L'itération directe des operateurs hermitiens et deux theories qui en dependent, Comment Math. Helv. vol. 15 (1942-1943) pp. 299-317. 
belonging to $\lambda_{1}^{\prime}$ if $\lambda_{1}^{\prime}$ is a characteristic number, but that $u^{i}$ converges weakly to 0 if $\lambda_{1}^{\prime}$ is in the continuous spectrum. If $\lambda_{1}^{\prime}$ is an isolated point of the spectrum $\delta(A)$ (and thus necessarily a characteristic number), and if the initial vector $x^{0}$ has a non-null projection on the characteristic manifold belonging to $\lambda_{1}^{\prime}$, then the vectors $x^{i}$ will converge (strongly) to a characteristic vector in this manifold. More generally, if no assumptions are made on $x^{0}$, then these convergence properties hold if $R$ is interpreted as the invariant closed linear manifold determined by $x^{0}, A x^{0}, A^{2} x^{0}, \cdots$.

By minimizing instead of maximizing $\mu$ at each step we obtain entirely analogous results for the infimum of the spectrum of $A$. We shall limit our discussion to the maximizing procedure. Further we shall suppose that $R$ is a real Hilbert space, not necessarily separable; there is no difficulty in extending the treatment to the complex case.

This paper is a generalization of an earlier one ${ }^{3}$ and some of the results obtained there will be used here.

2. An invariant subspace. We are dealing with a bounded selfadjoint operator $A$ on a real Hilbert space $R$, not necessarily separable, whose elements we call vectors. We let $F(\lambda)$ (continuous on the right) denote the spectral family of $A$ and $\mathrm{S}(A)$ the spectrum of $A$. Thus

$$
A=\int_{-\infty}^{\infty} \lambda d F(\lambda)=\int_{\mathcal{S}(\boldsymbol{A})} \lambda d F(\lambda) .
$$

A characteristic number (that is, a member of the point spectrum of $A$ ) is a real number $\lambda$ such that

$$
A y=\lambda y
$$

for some $y \neq 0$ in $R$; $y$ is a characteristic vector of $A$ belonging to $\lambda$. The characteristic vectors belonging to $\lambda$ determine a closed linear manifold, the characteristic manifold, which we designate by $\mathcal{L}(\lambda)$. This manifold is the projection manifold of $F(\lambda+)-F(\lambda-)=F(\lambda)$ $-F(\lambda-)$, where $F(\lambda+)$ and $F(\lambda-)$ denote, respectively, right- and left-hand limits.

We define $\mu(x)$ as in (1), and as before let

We have

$$
\lambda_{1}^{\prime}=\sup \delta(A) .
$$

$$
\mu(x) \leqq \lambda_{1}^{\prime}, \quad x \neq 0 \text { in } R .
$$

${ }^{8} \mathrm{~W}$. Karush, An iterative method for finding characteristic vectors of a symmetric matrix, Pacific Journal of Mathematics vol. 1 (1951) pp. 233-248. 
The proof is like that of (4) below. (We shall not require the stronger result (2).) Suppose now that $\lambda_{1}^{\prime}$ is a characteristic number. Let $\mathscr{B}^{\prime}$ be the set $\mathcal{S}(A)$ with $\lambda_{1}^{\prime}$ deleted, and put $\lambda_{2}^{\prime}=\sup \mathcal{B}^{\prime}$. Then we show that

$$
\mu(x) \leqq \lambda_{2}^{\prime}, \quad x \neq 0 \text { in } R \text { and orthogonal to } \mathcal{L}\left(\lambda_{1}^{\prime}\right) .
$$

For let $x$ be as described; we may assume $|x|=1$. Then

$$
\mu(x)=(A x, x)=\int_{S(A)} \lambda d|F(\lambda) x|^{2}=\int_{\mathcal{B}^{\prime}}+\int_{\lambda_{1}^{\prime}} .
$$

The second integral vanishes by the orthogonality of $x$; the first integral is dominated by $\lambda_{2}^{\prime}|x|^{2}=\lambda_{2}^{\prime}$, as desired. We shall not require the sharper result $\lambda_{2}^{\prime}=\sup \mu(x)(x$ as in (4)).

In the main we shall deal with the closed linear manifold determined by an initial non-null vector $x^{0}$ and the powers $A x^{0}$, $A^{2} x^{0}, \cdots$. We denote this manifold by $\mathfrak{F}$; symbolically

$$
\mathfrak{H C}=\left(x^{0}, A x^{0}, A^{2} x^{0}, \cdots\right) .
$$

$\mathfrak{H C}$ is a Hilbert space which is invariant under $A$, that is, $A \mathfrak{H C} \mathscr{H}$. We denote by $B$ the operator $A$ with domain restricted to $\mathcal{H C}$, that is, $B$ is a bounded self-adjoint operator on $x^{C}$ such that $B x=A x$ for $x$ in $\mathcal{F}$. It is not difficult to see that the spectral family $E(\lambda)$ of $B$ is obtained from the spectral family $F(\lambda)$ of $A$ by

$$
E(\lambda) x=F(\lambda) x
$$
$x$ in $\mathfrak{H C}$;

we omit the proof.

Clearly if a number is in the resolvent set of $A$ then it is in the resolvent set of $B$. Thus $\mathcal{S}(B) \subset \mathcal{S}(A)$. We let

$$
\lambda_{1}=\sup \delta(B) \text {. }
$$

The point spectrum of $B$ is obviously a subset of the point spectrum of $A$. Further, the characteristic numbers of $B$ are all simple. For let $\lambda$ be such a number and let $y_{1}, y_{2}$ be independent characteristic vectors of $B$ belonging to $\lambda$. Then some non-null linear combination $y$ of these vectors is orthogonal to $x^{0}$. From $B y=\lambda y$ it follows readily that $y$ is orthogonal to all powers $A^{j} x^{0}=B^{j} x^{0}, j=0,1,2, \cdots$. Hence, by (5), $y$ is orthogonal to $\mathfrak{H}$. But $y$ is in $\mathfrak{F}$. Hence $y=0$; contradiction. The next lemma shows how the characteristic vectors of $B$ are determined.

Lemma 1. Let $\lambda$ be a characteristic number of $A$ and $y(\lambda)$ be the projection of $x^{0}$ on the characteristic manifold $\mathcal{L}(\lambda)$ of $A$. Then $\lambda$ is a 
characteristic number of $B$ if and only if $y(\lambda) \neq 0$. If $y(\lambda) \neq 0$, then this vector is the unique characteristic vector of $B$ (apart from a scalar factor) belonging to $\lambda$.

Suppose $\lambda$ is characteristic for $B$ with characteristic vector $y \neq 0$ in Fe. If $y(\lambda)=0$, then $\mathcal{L}(\lambda)$ is orthogonal to $x^{0}$ and hence, by (5), to $\mathfrak{F}$. But $y$ is in $\mathcal{L}(\lambda)$. Hence $y=0$; contradiction.

Now suppose $y(\lambda) \neq 0$. By (6) and the definition of $y(\lambda)$,

$$
[E(\lambda)-E(\lambda-)] x^{0}=[F(\lambda)-F(\lambda-)] x^{0}=y(\lambda) .
$$

Thus $\lambda$ is a characteristic number of $B$ and $y(\lambda)$ is a characteristic vector. The uniqueness follows from the earlier remark that $\lambda$ is simple for $B$.

3. The iteration procedure. Consider a fixed integer $s>1$ and a fixed vector $x \neq 0$ in $R$. Define $A(x)$ as the finite-dimensional space

$$
A(x)=\left(x, A x, \cdots, A^{s-1} x\right),
$$

that is, the space spanned by the vectors indicated on the right. Suppose that the dimension of $\mathcal{A}(x)$ is $s$. The following statements in this paragraph were established in $\S \S 2$ and 3 of the previously cited paper by the author. The vectors $\xi_{0}, \xi_{1}, \cdots, \xi_{s-1}$ defined recursively by

$$
\begin{array}{rr}
\xi_{0}=x, \quad \xi_{1}=A \xi_{0}-\mu_{0} \xi_{0}, & \mu_{0}=\mu(x), \\
\xi_{j+1}=A \xi_{j}-\mu_{j} \xi_{j}-t_{j}^{2} \xi_{j-1}, & \mu_{j}=\mu\left(\xi_{j}\right), \quad t_{j}=\frac{\left|\xi_{j}\right|}{\left|\xi_{j-1}\right|}, \\
& j=1,2, \cdots, s-1,
\end{array}
$$

form an orthogonal basis for $\mathcal{A}(x)$. If the polynomials $p_{j}(\lambda)$ are defined by

$$
\begin{aligned}
p_{0}(\lambda) & =1, \quad p_{1}(\lambda)=\lambda-\mu_{0}, \\
p_{i+1}(\lambda) & =p_{j}(\lambda)\left(\lambda-\mu_{j}\right)-t_{i}^{2} p_{j-1}(\lambda), \quad j=1,2, \cdots, s-1,
\end{aligned}
$$

then

$$
\xi_{j}=p_{j}(A) x .
$$

The roots of $p_{j}(\lambda)$ are simple and real; let $\nu_{j}$ denote the maximum root. Then $\nu_{j}$ is the maximum of $\mu(x)$ for $x$ in $\left(x A x, \cdots, A^{i-1} x\right)$. In particular, letting $\nu=\nu_{s}$,

$$
\begin{gathered}
\nu=\max \mu(z), \quad z \neq 0 \text { in } \mathcal{A}(x), \\
\nu_{1} \leqq \nu_{2} \leqq \cdots \leqq \nu_{s}=\nu .
\end{gathered}
$$


Also

$$
p_{j}\left(\lambda^{\prime}\right) p_{j}(\lambda) \geqq 0, \quad\left|p_{j}\left(\lambda^{\prime}\right)\right| \geqq\left|p_{j}(\lambda)\right|, \quad \lambda^{\prime} \geqq \lambda \geqq \nu_{j} .
$$

Finally, there is a unique vector $x^{*}$ in $A(x)$ of the form $x+\eta$ with $(x, \eta)=0$ for which

$$
\mu\left(x^{*}\right)=\nu .
$$

It is given by

$$
x^{*}=x+\sum_{j=1}^{-1} \frac{p_{j}(\nu)}{\tau_{j}^{2}} \xi_{j}
$$

where

$$
\tau_{j}=t_{1} t_{2} \cdots t_{j}=\frac{\left|\xi_{j}\right|}{|x|} .
$$

Consider now the proposed iteration scheme. We construct a sequence of vectors $x^{0}, x^{1}, x^{2}, \cdots$ by choosing $x^{i+1}$ as that vector in $\mathcal{A}\left(x^{i}\right)$ of the form $x^{i}+\eta,\left(x^{i}, \eta\right)=0$, which maximizes $\mu(z), z$ in $\mathcal{A}\left(x^{i}\right)$. Assume for the moment that for each $i, \mathcal{A}\left(x^{i}\right)$ has dimension $s$. By (13) we have the explicit formula

$$
x^{i+1}=x^{i}+\sum_{j=1}^{s-1} \frac{p_{j}^{i}\left(\nu^{i}\right)}{\left(\tau_{j}^{i}\right)^{2}} \xi_{j}^{i}, \quad i=0,1,2, \cdots,
$$

where the superscript " $i$ " on the right has the obvious interpretation. It is clear that all vectors arising in this construction lie in the invariant space $\mathfrak{H C}$ of (5).

By (9) we have the alternative formula

$$
x^{i+1}=\left[I+\sum_{j=1}^{8-1} \frac{p_{j}^{i}\left(\nu^{i}\right)}{\left(\tau_{j}^{i}\right)^{2}} p_{j}^{i}(B)\right] x^{i},
$$

where $I$ is the identity operator. By the extremum property of $x^{i+1}$ in $A\left(x^{i}\right)$,

$$
\mu^{i+1} \geqq \mu^{i}, \quad \text { where } \mu^{i}=\mu\left(x^{i}\right) .
$$

From (15), (14) and the orthogonality of the $\xi_{j}^{i}$ for each $i$,

$$
\left|x^{i+1}\right|^{2}=\left|x^{i}\right|^{2}\left\{1+\sum_{j=1}^{\infty-1}\left[\frac{p_{j}^{i}\left(\nu^{i}\right)}{\tau_{j}^{i}}\right]^{2}\right\} .
$$

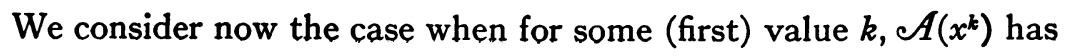


dimension less than $s$. Then $A\left(x^{k}\right)$ is invariant under $A$, and the vector $x^{k+1}$ maximizing $\mu(z)$ in this subspace is a characteristic vector of $A$. (This vector is given by (15) with " $s$ " replaced by the dimension of the subspace.) Since $A\left(x^{k+1}\right)=\left(x^{k+1}\right)$, it follows that the sequence $\left\{x^{i}\right\}$ has the constant value $x^{k+1}$ for $i \geqq k+1$. The iteration now becomes trivial, and the forthcoming proofs can be readily simplified to apply to this case. To save space we shall henceforth assume that for each $i$ the dimension of $\mathcal{A}\left(x^{i}\right)$ is $s$, and leave to the reader the appropriate modifications for the other case.

4. Convergence theorem. We establish the following convergence theorem.

TheOREM 1. For a given integer $s>1$ and initial vector $x^{0} \neq 0$, let $\left\{x^{i}\right\}$ be the sequence determined above. Let

Then

$$
u^{i}=\frac{x^{i}}{\left|x^{i}\right|}
$$

$$
\lim _{i \rightarrow \infty} \mu\left(x^{i}\right)=\lambda_{1}
$$

where $\lambda_{1}$ is given by (7). Further, if $\lambda_{1}$ is a characteristic number of $B$, then $u^{i}$ converges weakly (in $R$ ) to a characteristic vector of $B$ belonging to $\lambda_{1}$; if $\lambda_{1}$ is not a characteristic number of $B$, then $u^{i}$ converges weakly to 0 .

Proof. By (3), interpreted for $\mathfrak{H C}, \mu(x) \leqq \lambda_{1}$ for $x \neq 0$ in $\mathfrak{H C}$. Thus, from (17), the numbers $\mu^{i}$ have a limit $\bar{\mu} \leqq \lambda_{1}$. By definition $p_{2}^{i}(\lambda)$ $=\left(\lambda-\mu^{i}\right)\left(\lambda-\mu\left(\xi^{i}\right)\right)-\left|\xi^{i}\right|^{2} /\left|x^{i}\right|^{2}$, where

$$
\xi^{i}=B x^{i}-\mu^{i} x^{i}
$$

From (10) and (11) we have $p_{2}^{i}\left(\nu^{i}\right) \geqq 0$. Since $\mu^{i+1}=\nu^{i}$ by (12), we conclude that

$$
\frac{\left|\xi^{i}\right|^{2}}{\left|x^{i}\right|^{2}} \leqq\left(\mu^{i+1}-\mu^{i}\right)\left(\mu^{i+1}-\mu\left(\xi^{i}\right)\right) .
$$

Since the second factor on the right is bounded, the sequence on the left tends to 0 ; in fact

$$
\sum\left(t^{i}\right)^{2}<\infty, \quad t^{i}=\left|\xi^{i}\right| /\left|x^{i}\right| .
$$

From (19),

$$
\lim _{i \rightarrow \infty}\left(B u^{i}-\mu^{i} u^{i}\right)=0 .
$$


Suppose $\bar{\mu}<\lambda_{1}$. Choose $\bar{\lambda}$ so that $\bar{\mu}<\bar{\lambda}<\lambda_{1}$. Let $\Delta=I-E(\bar{\lambda}-)$. We note first that $\Delta x^{0} \neq 0$, i.e., $\Delta u^{0} \neq 0$. For, suppose $\Delta x^{0}=0$. Then from $\Delta B=B \Delta$ would follow $\Delta x=0$ for $x=B^{k} x^{0}, k=0,1,2, \cdots$, and thus $\Delta x=0$ for $x$ in $\mathcal{H}$, by (5). Hence $E(\bar{\lambda}-)=I$, so that $\sup \mathcal{S}(B)$ $\leqq \bar{\lambda}$, that is, $\lambda_{1} \leqq \bar{\lambda}$, a contradiction.

We put

$$
R_{j}^{i}=\frac{\left.p_{j}^{i}(\nu)^{i}\right)}{\left(\tau_{j}^{i}\right)^{2}}, \quad i=1,2, \cdots, s-1, i=0,1,2, \cdots
$$

By (16)

$$
x^{i+1}=\int_{-\infty}^{\lambda_{1}}\left[1+R_{1}^{i} p_{1}^{i}(\lambda)+\cdots+R_{s-1}^{i} p_{s-1}^{i}(\lambda)\right] d E(\lambda) x^{i} .
$$

Hence

$$
\left|\Delta x^{i+1}\right|^{2}=\int_{\bar{\lambda}}^{\lambda_{1}}\left[1+R_{1}^{i} p_{1}^{i}(\lambda)+\cdots+R_{s-1}^{i} p_{s-1}^{i}(\lambda)\right]^{2} d\left|E(\lambda) x^{i}\right|^{2} .
$$

By (11), the quantity in brackets is an increasing function of $\lambda$ in the range of integration. Thus

$$
\left|\Delta x^{i+1}\right|^{2} \geqq\left[1+R_{1}^{i} p_{1}^{i}(\bar{\lambda})+\cdots+R_{s-1}^{i} p_{s-1}^{i}(\bar{\lambda})\right]^{2}\left|\Delta x^{i}\right|^{2} .
$$

From (18)

$$
\frac{\left|x^{i+1}\right|^{2}}{\left|x^{i}\right|^{2}}=1+R_{1}^{i} p_{1}^{i}\left(\nu^{i}\right)+\cdots+R_{-1}^{i} p_{-1}^{i}\left(\nu^{i}\right)
$$

so that, by (11),

$$
\left|\Delta u^{i+1}\right|^{2} \geqq \frac{1+R_{1}^{i} p_{1}^{i}(\bar{\lambda})+\cdots+R_{s-1}^{i} p_{s-1}^{i}(\bar{\lambda})}{1+R_{1}^{i} p_{1}^{i}\left(\nu^{i}\right)+\cdots+R_{s-1}^{i} p_{s-1}^{i}\left(\nu^{i}\right)}\left|\Delta u^{i}\right|^{2} \geqq\left|\Delta u^{i}\right|^{2} .
$$

On the other hand

$$
\begin{aligned}
& \left|B u^{i}-\mu^{i} u^{i}\right|^{2}=\int_{-\infty}^{\lambda_{1}}\left(\lambda-\mu^{i}\right)^{2} d\left|E(\lambda) u^{i}\right|^{2} \\
& \quad \geqq \int_{\bar{\lambda}}^{\lambda_{1}}\left(\lambda-\mu^{i}\right)^{2} d\left|E(\lambda) u^{i}\right|^{2} \geqq(\bar{\lambda}-\bar{\mu})^{2}\left|\Delta u^{i}\right|^{2} .
\end{aligned}
$$

By (21) it follows that $\left|\Delta u^{i}\right| \rightarrow 0$, a contradiction to (23) and the earlier result $\left|\Delta u^{0}\right| \neq 0$. This establishes the first conclusion of the theorem. 
Suppose now that $\lambda_{1}$ is not a characteristic number of $B$. If $u^{i}$ does not converge weakly to 0 (in $\mathcal{H}$ ), then a subsequence $u^{k}$ in $\mathcal{H C}$ must have a weak limit $\bar{u} \neq 0$ (in $\mathfrak{H}$ ), since $\left|u^{i}\right|=1$. Thus $B u^{k}-\mu^{k} u^{k}$ converges weakly to $B \bar{u}-\lambda_{1} \bar{u}$. But this sequence converges (strongly) to zero by (21). Hence $B \bar{u}-\lambda_{1} \bar{u}=0$, contrary to $\lambda_{1}$ not being characteristic. Hence $u^{i}$ converges weakly to 0 in $\mathcal{H C}$, and hence in the original space $R$.

Finally, suppose $\lambda_{1}$ is characteristic for $B$. Let $y_{1}$ be a characteristic vector of $B$ belonging to $\lambda_{1}$. By Lemma $1,\left(x^{0}, y_{1}\right) \neq 0$ and $\lambda_{1}$ is simple. We normalize $y_{1}$ so that $\left|y_{1}\right|=1,\left(x^{0}, y_{1}\right)>0$. Any solution of $B z=\lambda_{1} z$ in $\mathcal{H C}$ is a multiple of $y_{1}$. By (16) and (22),

$$
\begin{aligned}
& \left(x^{i+1}, y_{1}\right)=\left[1+R_{1}^{i} p_{1}^{i}\left(\lambda_{1}\right)+\cdots+R_{s-1}^{i} p_{s-1}^{i}\left(\lambda_{1}\right)\right]\left(x^{i}, y_{1}\right), \\
& \left(u^{i+1}, y_{1}\right) \geqq \frac{1+R_{1}^{i} p_{1}^{i}\left(\lambda_{1}\right)+\cdots+R_{s-1}^{i} p_{s-1}^{i}\left(\lambda_{1}\right)}{1+R_{1}^{i} p_{1}^{i}\left(\nu^{i}\right)+\cdots+R_{s-1}^{i} p_{s-1}^{i}\left(\nu^{i}\right)}\left(u^{i}, y_{1}\right) \geqq\left(u^{i}, y_{1}\right),
\end{aligned}
$$

using (11). Hence $\left(u^{i}, y_{1}\right) \rightarrow L>0$. Now suppose $\bar{u}$ is any weak limit (in $\mathfrak{F C}^{\mathrm{C}}$ ) of a subsequence $u^{k}$ of $u^{i}$. As in the above paragraph, $B \bar{u}$ $=\lambda_{1} \bar{u}$. Hence $\bar{u}=l y_{1}$. But $L=\lim _{k \rightarrow \infty}\left(u^{k}, y_{1}\right)=\left(l y_{1}, y_{1}\right)=l$. Hence $l=L$, $\bar{u}=L y_{1}$, independently of $\bar{u}$. This establishes that $L y_{1}$ is the weak limit in $\mathfrak{H}$, and thus in $R$, of $u^{i}$, and completes the proof of the theorem.

In the following corollary we relate the preceding result directly to the original operator $A$.

Corollary to Theorem 1. If $F(\lambda) x^{0} \neq x^{0}$ for $\lambda<\lambda_{1}^{\prime}$, where $\lambda_{1}^{\prime}$ $=\sup S(A)$, then $\mu^{i}$ converges to $\lambda_{1}^{\prime}$. If $\lambda_{1}^{\prime}$ is characteristic for $A$ and $x^{0}$ has a non-null projection on the characteristic manifold $\mathcal{L}$ of $A$ belonging to $\lambda_{1}^{\prime}$, then $u^{i}$ converges weakly to a characteristic vector in $\mathcal{L}$.

Proof. Consider the first statement of the corollary. We have $\lambda_{1} \leqq \lambda_{1}^{\prime}$. Suppose $\lambda_{1}<\lambda_{1}^{\prime}$. Then by (6)

$$
x_{0}=\int_{-\infty}^{\lambda_{1}} d E(\lambda) x^{0}=\int_{-\infty}^{\lambda_{1}} d F(\lambda) x^{0}=F\left(\lambda_{1}\right) x^{0},
$$

contrary to hypothesis. Hence $\lambda_{1}=\lambda_{1}^{\prime}$, and the conclusion follows from Theorem 1 . The second statement is a consequence of Lemma 1 and Theorem 1.

\section{Convergence theorem for $\lambda_{1}$ isolated.}

Lemma 2. Let $\lambda_{1}$ be an isolated point of $S(B)$. Then the unit vectors $u^{i}$ converge to the characteristic vector of $B$ belonging to $\lambda_{1}$. 
Proof. Since $\lambda_{1}$ is isolated, it is a characteristic number for $B$. Let $y_{1}$ be the corresponding characteristic vector, normalized so that $\left|y_{1}\right|=1$ and $\left(x^{0}, y_{1}\right)>0$. In the last paragraph of the proof of Theorem 1 we showed that $u^{i}$ converged weakly to $L y_{1}$, where $L=\lim _{i \rightarrow \infty}\left(u^{i}, y_{1}\right)$ $>0$. We now show that $u^{i}$ converges to $y_{1}$.

Write $u^{i}=y^{i}+z^{i}$ with $y^{i}$ a multiple of $y_{1}$ and $z^{i}$ in $\mathcal{F}$ orthogonal to $y_{1}$. Let

$$
\lambda_{2}=\sup \mathbb{B},
$$

where $B$ is $S(B)$ with $\lambda_{1}$ deleted. Then $\lambda_{2}<\lambda_{1}$, and by (4), interpreted for $\mathcal{H}$, we have $\mu\left(z^{i}\right) \leqq \lambda_{2}$. Now $\left(y^{i}, y_{1}\right)=\left(u^{i}, y_{1}\right) \rightarrow\left(L y_{1}, y_{1}\right)=L$. Hence $y^{i}=\left(y^{i}, y_{1}\right) y_{1}$ converges to $L y_{1}$.

Using the definition (1) of $\mu$ we find

$$
\begin{aligned}
\mu^{i} & =\mu\left(u^{i}\right)=\left(B u^{i}, u^{i}\right)=\mu\left(y^{i}\right)\left|y^{i}\right|^{2}+\mu\left(z^{i}\right)\left|z^{i}\right|^{2} \\
& =\lambda_{1}\left(1-\left|z^{i}\right|^{2}\right)+\mu\left(z^{i}\right)\left|z^{i}\right|^{2} .
\end{aligned}
$$

Thus

$$
\lambda_{1}-\mu^{i}=\left(\lambda_{1}-\mu\left(z^{i}\right)\right)\left|z^{i}\right|^{2} \geqq\left(\lambda_{1}-\lambda_{2}\right)\left|z^{i}\right|^{2} .
$$

Since $\mu^{i} \rightarrow \lambda_{1}$ by Theorem 1 , it follows that $z^{i}$ converges to 0 . From $u^{i}=y^{i}+z^{i}$ we now deduce that $u^{i}$ converges to $L y_{1}$. Since $\left|u^{i}\right|=\left|y_{1}\right|$ $=1$, we must have $L=1$. This completes the proof.

Our goal is to replace " $u^{i}$ " by " $x$ " in the above lemma. For this it is clearly sufficient to show that the increasing lengths $\left|x^{i}\right|$ (see (18)) are bounded. To this end we introduce the next lemma. (As stated at the end of $\S 3$, we are assuming that for each $i$ the vectors $x^{i}, A x^{i}, \cdots$, $A^{8-1} x^{i}$ are independent. As shown there, if this is not the case then the sequence $\left\{x^{i}\right\}$ is eventually constant, and obviously the lengths $\left|x^{i}\right|$ converge.)

Lemma 3. Let $\lambda_{1}$ be an isolated point of $S(B)$. There is a constant $K$, independent of $i$ and $j$, such that for $i$ sufficiently large,

$$
\left|p_{j}^{i}\left(\nu^{i}\right)\right| \leqq K\left(\tau_{j}^{i}\right)^{2}, \quad j=1,2, \cdots, s-1 .
$$

We shall not give the details of the proof; they can be found in the proof of a similar result in the previously cited paper by the author. One first establishes, as in Lemma 1 of the earlier paper, that

$$
\lambda_{1}-\mu(x) \leqq \frac{1}{\mu(x)-\lambda_{2}} \cdot \frac{|B x-\mu(x) x|^{2}}{|x|^{2}}
$$

for every $x$ in $\mathcal{H C}$ with $\mu(x)>\lambda_{2}, \lambda_{2}$ as in (24). The lemma is then established by an argument like that of Lemma 2 of the earlier paper. 
THEOREM 2. Let $\lambda_{1}$ be an isolated point of $S(B)$. Then the vectors $x^{2}$ of Theorem 1 converge to the characteristic vector of $B$ belonging to $\lambda_{1}$.

Proof. We use (18). By a standard theorem on infinite products the numbers $\left|x^{i}\right|^{2}$ will converge if each of the series $\sum_{i=0}^{\infty}\left[p_{j}^{i}\left(\nu^{i}\right) / \tau_{j}^{i}\right]^{2}$, $j=1,2, \cdots, s-1$, converges. By Lemma 3 this will occur if each of the series $\sum_{i=0}^{\infty}\left(\tau_{j}^{i}\right)^{2}$ converges. By (20), this series converges for $j=1$. By (8),

$$
\left|\xi_{j+1}^{i}\right| \leqq K_{1}\left|\xi_{j}^{i}\right|+\left(t_{j}^{i}\right)^{2}\left|\xi_{j-1}^{i}\right|
$$

Hence

$$
t_{j+1}^{i} \leqq K_{1}+i_{j}^{i}
$$

Since $t_{1}^{i}$ is bounded, it follows that there is a constant $K_{2}$ such that $t_{j+1}^{i} \leqq K_{2}$ for all $i$ and for $j=1,2, \cdots, s-2$. Hence by (14),

$$
\sum_{i}\left(\tau_{j+1}^{i}\right)^{2}=\sum_{i}\left(\tau_{j}^{i}\right)^{2}\left(t_{j+1}^{i}\right)^{2} \leqq K_{2}^{2} \sum_{i}\left(\tau_{j}^{i}\right)^{2} .
$$

This establishes the convergence of all the series and completes the proof.

By Lemma 1 and Theorem 2 we obtain the following result.

Corollary to Theorem 2. Let $\lambda_{1}^{\prime}=\sup S(A)$ be an isolated point of $\mathcal{S}(A)$. If $x^{0}$ is not orthogonal to the characteristic manifold of $A$ belonging to $\lambda_{1}^{\prime}$, then $x^{i}$ converges to a characteristic vector in this manifold.

University of Chicago and

National Bureau of Standards 